\title{
Surgical Management of Idiopathic Perianal Fistulas: A Systematic Review and Meta-Analysis
}

\author{
Xinyi Cherry Cheung ${ }^{a}$ Tom Fahey $^{b}$ Ailin C. Rogers ${ }^{b}$ \\ John Hogeland Pemberton ${ }^{c}$ Dara Oliver Kavanagh ${ }^{a, d}$ \\ ${ }^{a}$ Department of Surgery, Tallaght University Hospital, Dublin, Ireland; ${ }^{b}$ Department of Postgraduate Studies, \\ RCSI, Dublin, Ireland; 'Division of Colon \& Rectal Surgery, Mayo Clinic, Rochester, MN, USA; ${ }^{\text {dDepartment of }}$ \\ Surgical Affairs, RCSI, Dublin, Ireland
}

\section{Keywords}

Fistula · Plug · Fibrin glue · Crohn's disease .

Cryptoglandular fistula

\begin{abstract}
Background: Perianal fistula is a common colorectal condition with an incidence of 9 per 100,000. Many surgical treatments exist, all aiming to eliminate symptoms with minimal risk of recurrence and impact upon continence. Despite extensive evaluation of the therapeutic modalities, no clear consensus exists as to what is the gold standard approach. This systematic review aimed to examine all available evidence pertaining to the surgical management of perianal fistulas. Primary outcomes examined were recurrence and incontinence. Summary: This study was conducted according to PRISMA guidelines. Primary outcomes were analyzed for each group and expressed as pooled odds ratio with confidence intervals of $95 \% .687$ studies were identified from which 28 relevant studies were included. There was no significant difference in rates of incontinence identified between various surgical approaches. Glues and plugs show higher recurrence rates. Newer treatments continue to emerge with promise but lack supporting evidence of ben-
\end{abstract}

efit over conventional therapies. Key Messages: While we await more robust randomized data, we will continue to proceed cautiously trying to offset the benefits of fistula healing against the inherent risk of altered continence.

(c) 2021 S. Karger AG, Basel

\section{Introduction}

Perianal fistula is one of the most common colorectal conditions impacting significantly on patients' quality of life with a reported incidence of 9 per 100,000 [1]. It is a very common initial manifestation of perianal Crohn's disease (CD) ( 30\%). Several authors have proposed the cryptoglandular theory as the likely aetiology in the absence of perianal CD [2-4]. This is the most plausible explanation although it lacks supporting evidence. While fistulas are rarely life threatening, they can be very debilitating and socially embarrassing.

Various treatment options are available with the aim of eliminating the tract while achieving low recurrence rates and having minimal impact upon continence. Incontinence is a spectrum of illness ranging from sporadic flatus incontinence to overt soiling with social isolation karger@karger.com www.karger.com/dsu

(c) 2021 S. Karger AG, Base

Karger"
Dara Oliver Kavanagh

Department of Surgical Affairs

RCSI, Royal College of Surgeons in Ireland

123 St Stephens Green, IE Dublin 2 (Ireland)

darakavanagh@rcsi.com 
and severe impact on quality of life. Setons are one of the oldest means of treating perianal fistulas with cutting setons to externalize trans-sphincteric fistula tracts and draining setons to control sepsis within fistula tracts especially in patients with $\mathrm{CD}$ [5]. A variety of materials have been used including non-absorbable silk sutures or rubber vascular loops $[4,6,7]$. The latter rarely induce healing $(<10 \%)$. While relatively easy to perform, setons are associated with a reported incontinence rate of $20-67 \%[8,9]$. Advancement flaps - mucosal or endorectal - involve complete excision of the tract and the closure of the subsequent defect with a raised rectal mucomuscular flap. Endorectal flaps are variations in which the mucosal flap is raised in a submucosal plane superior to the internal defect. Reported success rates for endorectal flaps range from 55 to $98 \%$ [6].

Fistulotomy involves laying open of the fistula tract by incising onto the tract. Success rates of $90 \%$ have been reported, but a variable incontinence rate of $5-30 \%$ is also described [7]. The addition of marsupialization has expedited healing times in select series. Fistulectomy involves complete excision of the fistula tract including its openings. Wound sizes are larger with fistulectomy, and hence healing time is prolonged [6]. Marsupialization can expedite healing. Ligation of intersphincteric fistula tract (LIFT) procedure is a relatively novel therapy first introduced in 2007 [10]. It involves accessing the fistula tract through the intersphincteric space and interrupting and ligating both ends of the fistulous tract. Modifications of this include the addition of a mucosal advancement flap, plug, or biograft mesh following interruption of the tract $[6,7]$. This approach has reported success between 50 and $80 \%$ [6].

Different types of glues to obliterate the fistula tract have been tried. The main issue with glues is high recurrence rates. Successful healing rates vary between 31 and $85 \%[11,12]$. Similar to glues, fistula plugs work by obliterating the tract $[4,6]$. Incontinence rates are low, but results are variable with success rates of 14-87\% [13]. Autologous stem cells derived from adipose tissue have recently been utilized in the treatment of perianal fistulas. Stem cells are extracted and purified from adipose tissue obtained by liposuction [14].

Despite continuing research and novel therapies, there remains a lack of consensus as to what is the best management plan for patients with perianal fistulae not related to $\mathrm{CD}$. The strongest evidence to date is presented by the most recent Cochrane review in 2010 which concludes that there is no difference in recurrence rates between the various treatments, but flaps and glues may have lower incontinence rates [15]. Novel treatments such as LIFT

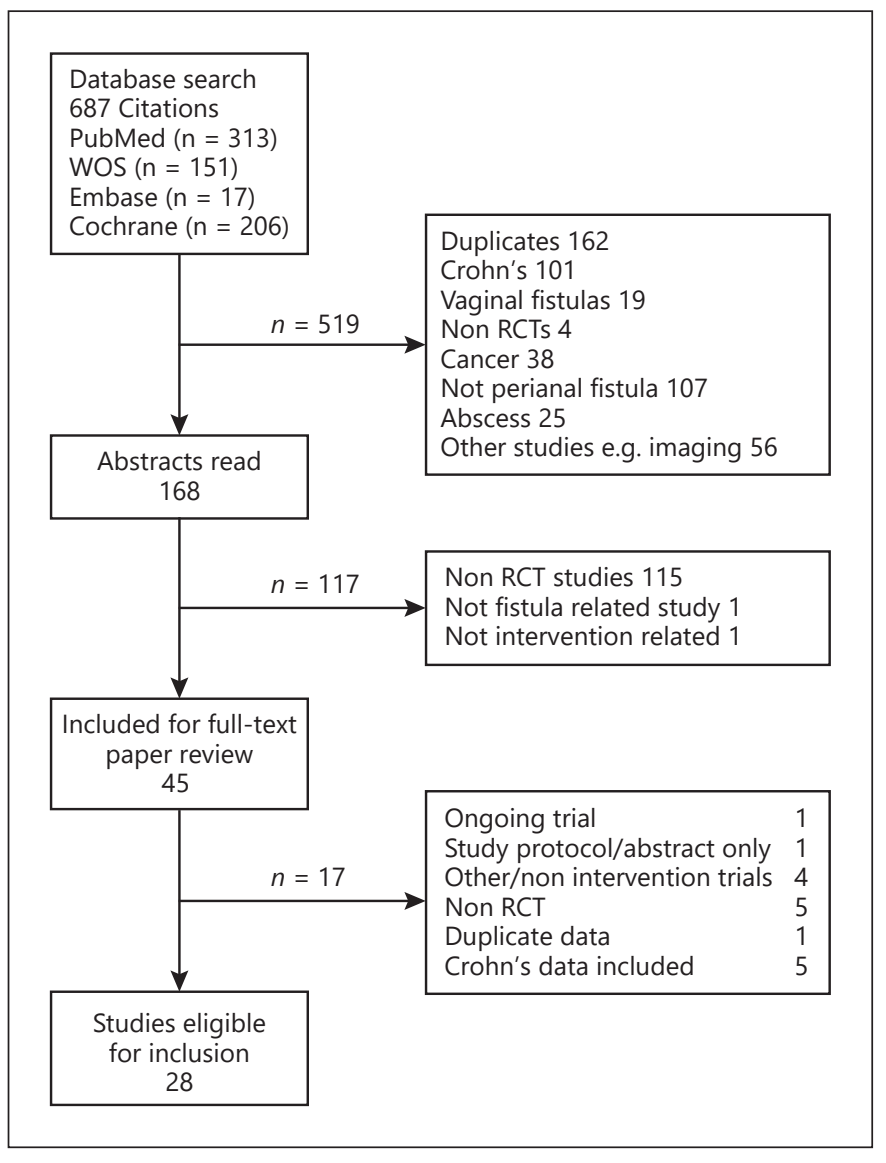

Fig. 1. Flowchart of eligible studies including included and excluded trials.

and stem cell injection have shown promise based on early data but were not included in the 2010 Cochrane review. The aim of this study is to conduct a systematic review of all the current available evidence on the surgical management of non-Crohn's-related perianal fistulas, comparing their outcomes based on rates of recurrence and incontinence.

\section{Methods}

This meta-analysis was conducted in accordance with the preferred reporting items for systematic reviews and meta-analyses (PRISMA) guidelines (see online suppl. Appendix; for all online suppl. material, see www.karger.com/doi/10.1159/000512652). Approval from an ethics committee was not required for this study. An extensive search strategy was devised in order to maximize the capture of relevant studies and perform a highly sensitive search (online suppl. Appendix). The search strategy was applied to 4 major online databases: PubMed, Embase, Web of Science, and the Cochrane Central Register of Controlled Trials. Reference lists of included studies were also reviewed to identify any other potential studies. 


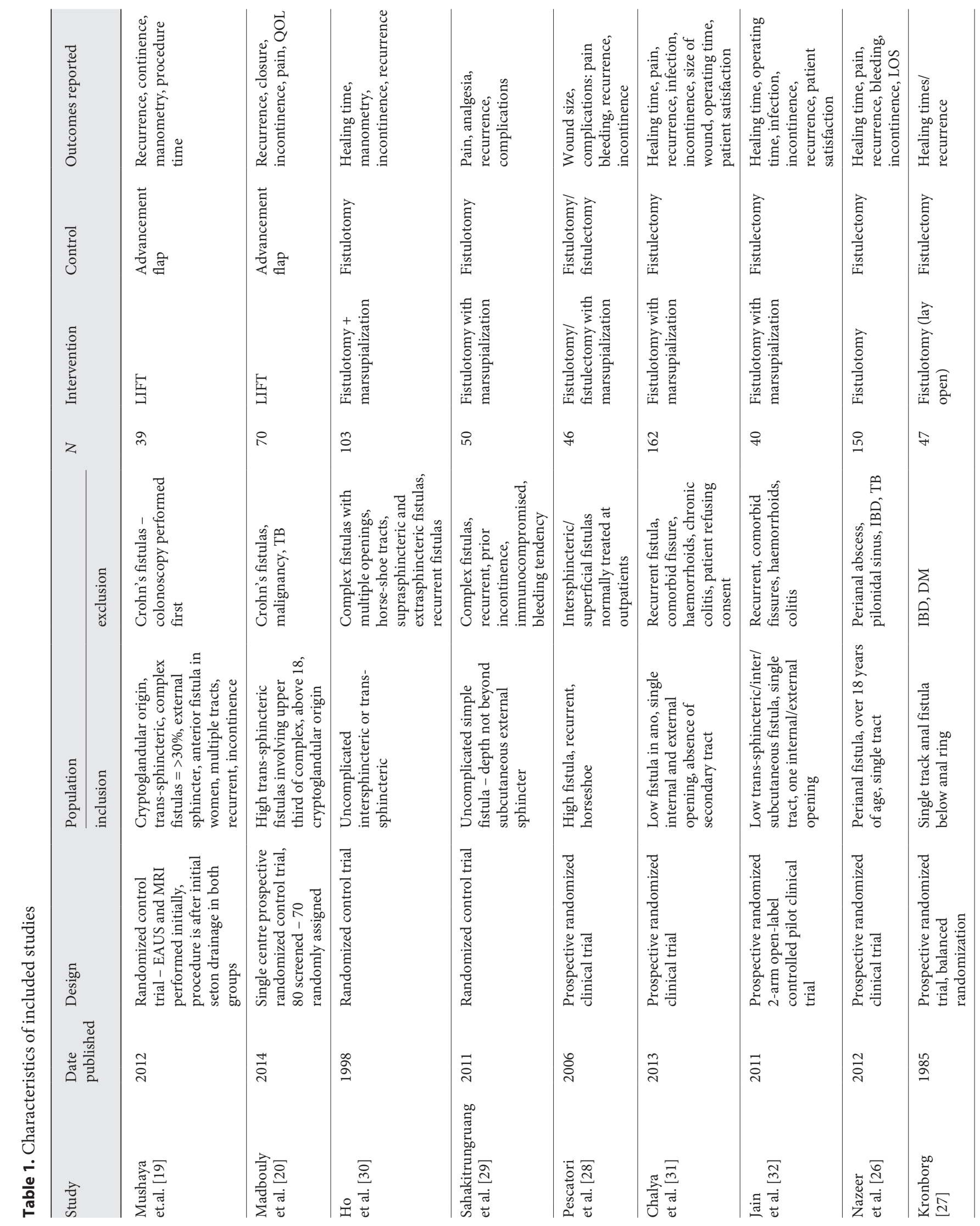




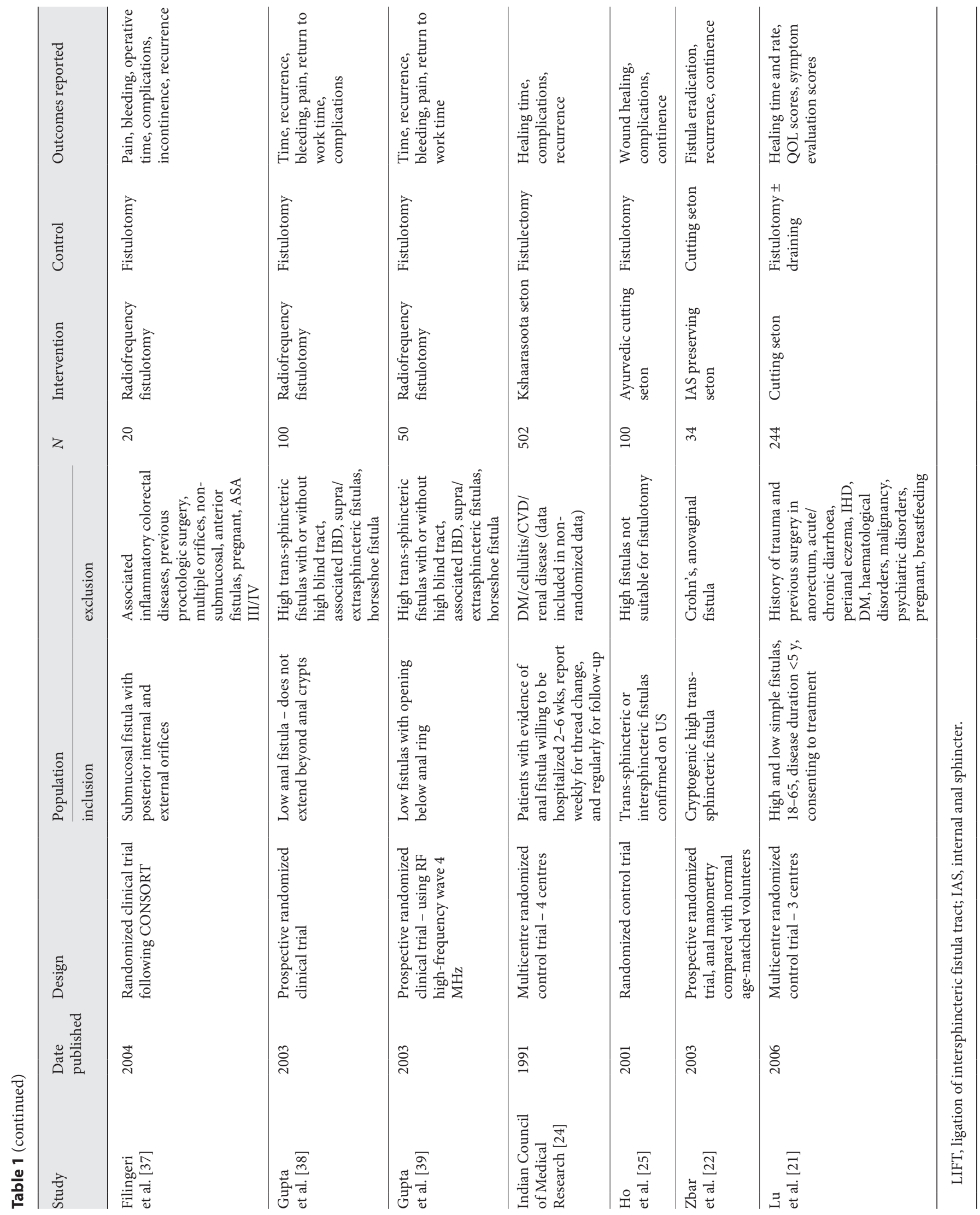




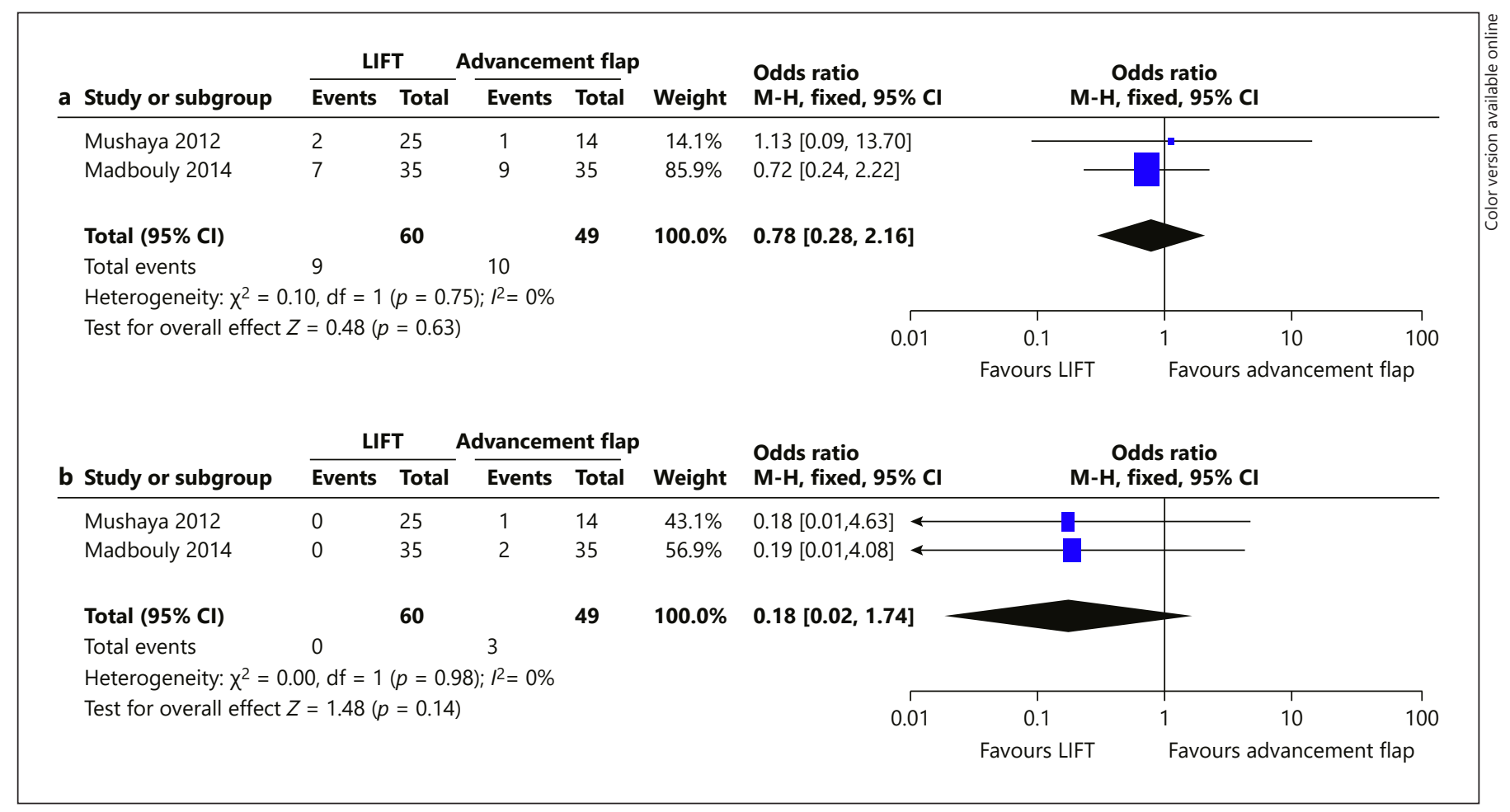

Fig. 2. Comparison of LIFT versus advancement flap in relation to the primary outcomes recurrence (a) and faecal incontinence (b) with funnel plots. LIFT, ligation of intersphincteric fistula tract.

\section{Eligibility Criteria}

The target population of this study was adult patients (over 18 years of age) with perianal fistulas. We focussed upon perianal fistulas, the aetiology of which is cryptogenic in nature. All randomized control trials on surgical management of cryptogenic perianal fistulas were included. The search was not confined to Englishlanguage articles.

Fistulas related to inflammatory bowel disease, in particular $\mathrm{CD}$, have been excluded as concomitant medical management with immunomodulatory therapies form the mainstay of treatment for these types of fistulas [16]. Fistulas associated with malignancy or hidradenitis suppurativa together with rectovaginal fistulas are considered separate entities with different treatment modalities and are also excluded from this study (online suppl. Appendix).

\section{Search}

Two independent reviewers applied the inclusion/exclusion criteria to the citation list (C.C. and A.R.). Any disagreement was resolved by a third independent reviewer (D.K.). We appraised 46 full-text manuscripts after initial exclusion, and 28 were eligible for inclusion. Abstracts and full manuscripts that were in a foreign language were translated by an online translating tool (Google Translate) by one of the reviewers (C.C.) [17].

\section{Dataset}

Data from included studies were extracted and inputted onto an electronic spreadsheet in excel format. Defined outcome mea- sures were extracted for meta-analysis. Data were grouped according to the comparative groups in each study to allow pooling of data from studies examining the same comparative groups.

\section{Outcomes}

The primary outcomes for meta-analysis in this study were fistula recurrence and faecal incontinence. Raw data for each outcome were extracted and analyzed. Rates of recurrence and incontinence were examined by recording the total number of patients in each comparison group (intervention and control) and the total events in each group.

\section{Statistical Analysis}

RevMan version 5.3.5 (Cochrane Collaboration, Copenhagen, Denmark) was used to complete statistical analyses for the dataset. For each comparison group, odds ratio of the dichotomous outcomes of (1) recurrence and (2) incontinence were pooled where possible. Mantel-Haenszel fixed-effects models were used in all comparison groups. Odds ratios of compared outcomes were described with $p$ values $<0.05$ as statistically significant. We accepted a confidence interval of $95 \%$ for all outcomes. Forest plots were drawn for each analysis. The $I^{2}$ statistic was used to assess statistical heterogeneity objectively. The potential for publication bias was evaluated by visually inspecting funnel plots where possible. Quality of the included studies was measured using the Jadad scale for the assessment of quality of reports in randomized clinical trials [18] (online suppl. Appendix). A score of $>2$ indicates good methodological quality [18]. 


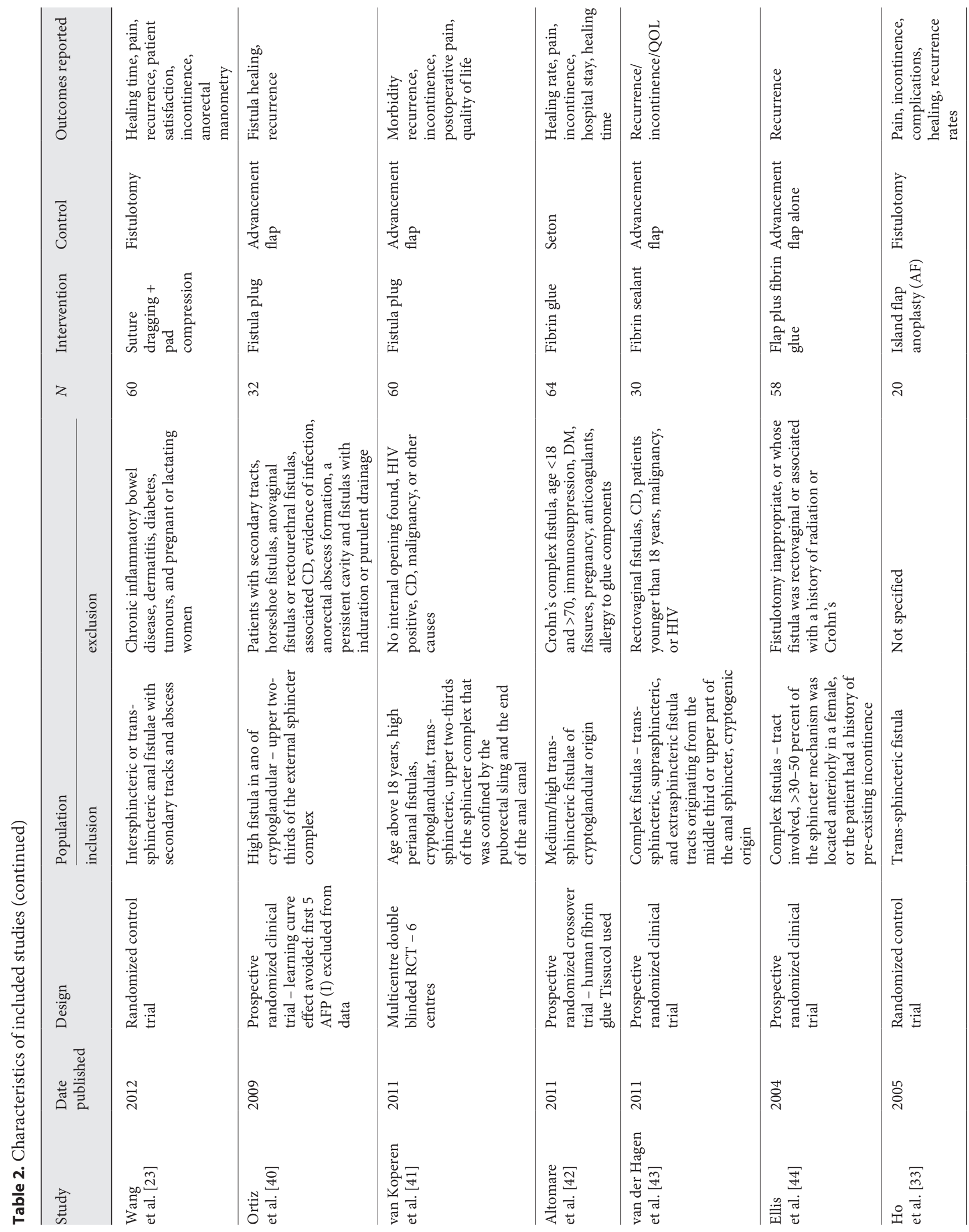




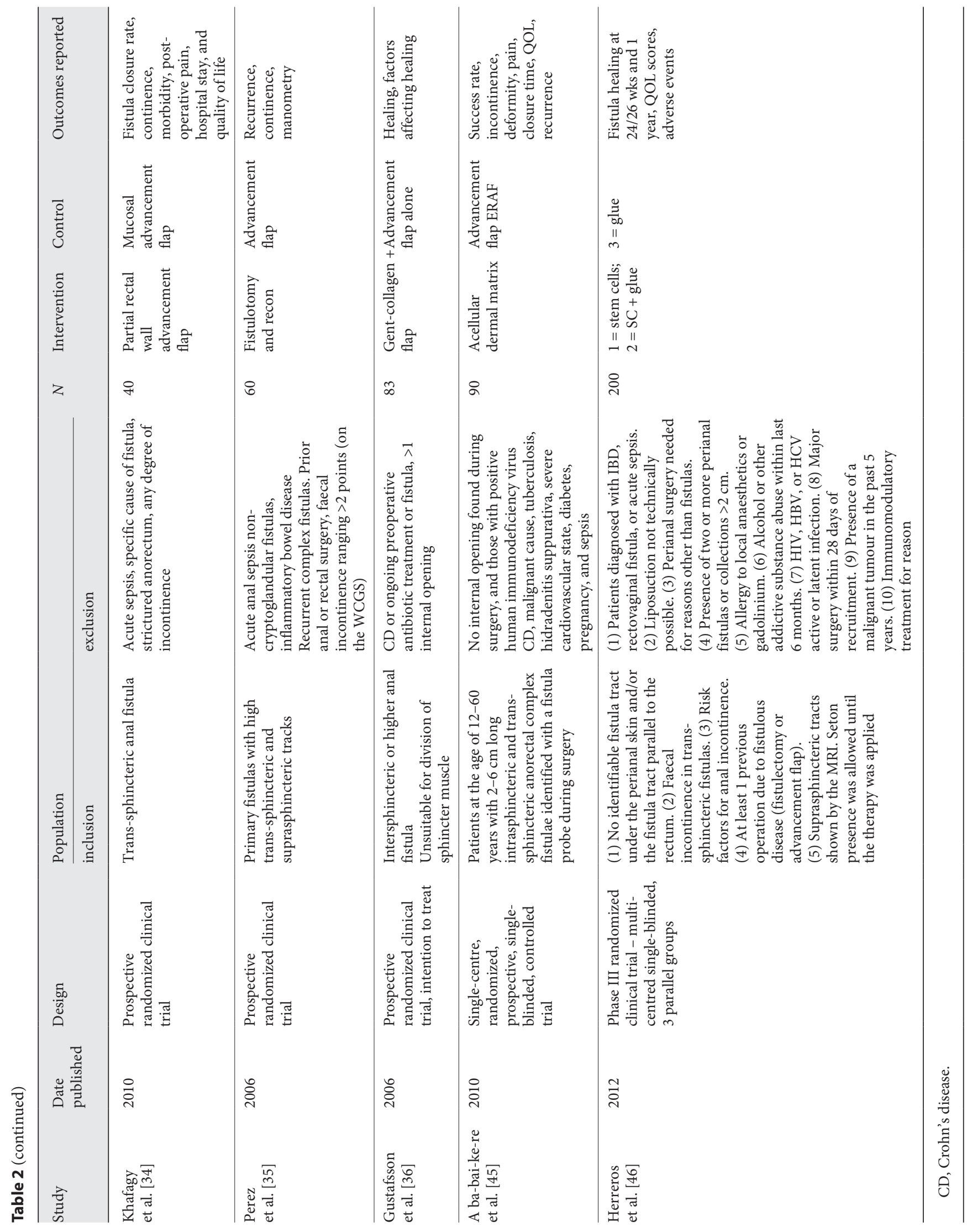




\section{Results}

We identified 687 studies. Figure 1 (flowchart) summarizes the results of the search. A total of 28 studies were included in this review. As the topic of perianal fistulas and its surgical treatment is so diverse, we found that the 28 included studies had a range of different comparison groups, and thus pooling of data from all 28 studies was not possible. We divided the 28 studies into 9 different sections with similar comparison groups in order to analyze pooled data where possible. The characteristics of the 28 included studies are shown in Tables 1 and 2, and the main results from the studies are presented in Tables 3 and 4.

Twenty six out of 28 included studies reported on recurrence rates with 7 out of 26 reporting no recurrence in either intervention or control intervention groups (online suppl. Tables 1,2). Data from 19 studies were suitable for analysis. Seventeen comparative groups were analyzed. Twenty out of 28 studies reported on incontinence as a primary outcome. Fifteen of these studies provided data for possible analysis (online suppl. Tables 1,2). In all studies that reported on incontinence, clinical follow-up occurred at the outpatient setting. There was no objective measurements of continence performed.

\section{LIFT Procedure}

Two RCTs were included in our analysis $[19,20]$. Both used advancement flap as a control group and found no statistically significant difference in recurrence rates. Pooled odds ratio for recurrence is 0.78 with a $95 \%$ confidence interval of $0.28-2.15, p=0.63$ (Fig. 2a). There was no significant heterogeneity evident with an $I^{2}$ of $0 \%$. Comparison between the groups for post-operative incontinence also showed no significant difference. Pooled odds ratio for incontinence is $0.18,95 \%$ CI: $0.02-1.74$, $p=0.14$. There is no significant heterogeneity evident with an $I^{2}$ of $0 \%$ (Fig. 2b). Pain scores in the LIFT group, 0 and 3.1, were significantly lower than in the advancement flap group, 1 and $4.8(p=0.017, p=0.002)$.

\section{Setons}

Six included studies examined the use of different types of setons for treating perianal fistulas. Three studies examined the use of different cutting setons in transsphincteric fistulas $[22,23]$. There was a single RCT, and hence meta-analysis of pooled data was not possible.

Internal anal sphincter (IAS) preserving seton involves rerouting of the fistula tract in the intersphincteric space. In a study that compared IAS preserving seton versus

Surgical Management of Perianal Fistulas conventional cutting seton [22], there was no difference in recurrence rates (OR 1.88, 95\% CI: $0.15-22.88, p=$ 0.62 ). Incontinence rates between IAS preserving seton and conventional cutting seton were not shown to be significant (OR 0.41, 95\% CI: 0.03-5.03, $p=0.49$ ) [22].

\section{Fistulotomy versus Fistulectomy}

Two studies compared fistulotomy with fistulectomy in simple low fistulas [26, 27]. Nazeer et al. [26] reported no recurrence or incontinence in both groups after a 10-month follow-up (no statistics were provided). Data from the study by Kronborg [27] alone were analyzed here. Three out of 24 from the fistulotomy group recurred compared to 2 out of 21 from the fistulectomy group, giving an OR of 1.36 (95\% CI: $0.20-9.02, p=0.75)$. This difference is not statistically significant (online suppl. Fig. 1a). A single case of post-operative incontinence occurred in the fistulotomy group $(n=24)$ compared to 3 cases in the fistulectomy group $(n=21)$. There was no significant difference in incontinence between the groups with an OR of 0.26 (95\% CI: 0.02-2.72, $p=0.26$ ) (online suppl. Fig. 1b). Kronborg [27] also reported a significant decrease in healing time in the fistulotomy group with a median healing time of 34 days versus 41 days in the fistulectomy group $(p<0.02)$.

\section{Marsupialization of the Fistula Wound}

Fistulotomy and marsupialization ultimately results in a decreased wound size. Five studies examining marsupialization were included in our analysis [28-32]. Two studies compared it with fistulotomy alone $[29,30]$. Two studies used fistulectomy as controls [31,32]. One study used fistulotomy and/or fistulectomy as the control group [28].

Marsupialization versus Fistulotomy Alone

Three studies were included $[28,30]$. There was no difference in recurrence rates. Pooled OR is $0.84,95 \%$ CI: $0.21-3.34, p=0.80$ (online suppl. Fig. 2a). There is no evidence of significant heterogeneity $\left(I^{2} 0 \%\right)$. Analysis of incontinence in these groups gave an OR of 0.5 (95\% CI: $0.14-1.72, p=0.27$; online suppl. Fig. 2 b). Post-operative continence is not significantly different between the comparison groups. Heterogeneity is insignificant with an $I^{2}$ of $0 \%$.

Marsupialization versus Fistulectomy

Three studies examined marsupialization versus fistulectomy $[28,31,32]$. Two studies reported no cases of recurrence or incontinence. Data from the study by Pesca- 
Table 3. Summary of overall results

\begin{tabular}{|c|c|c|c|c|c|}
\hline Study & $\begin{array}{l}\text { Procedures } \\
\text { compared }\end{array}$ & $\begin{array}{l}\text { Control } \\
\text { group }\end{array}$ & Results & & Main findings \\
\hline $\begin{array}{l}\text { Mushaya } \\
\text { et al. [19] }\end{array}$ & LIFT & $\begin{array}{l}\text { Advancement } \\
\text { flap }\end{array}$ & $\begin{array}{l}\text { Operative time: LIFT median } 10 \mathrm{~min} \\
\text { AF } 42.5, p<0.0011 \\
\text { Satisfaction scores: LIFT 9.5; AF } 8.07 \\
p=<0.001 \\
\text { Pain score: LIFT } 0 \text {, AF } 1, p=0.017\end{array}$ & $\begin{array}{l}\text { Time to } N \text { activities: LIFT } 1.0 \\
\text { wks, AF } 2.0 \text { wks, } p=0.16 \\
\text { Recurrence: AF } 1 / 14, \text { LIFT } \\
2 / 25 \\
\text { Incontinence: AF } 1 / 14, \text { LIFT } \\
0 / 25\end{array}$ & $\begin{array}{l}\text { LIFT is safe and simple } \\
\text { Shorter to perform and } \\
\text { patients return to work } \\
\text { sooner } \\
\text { No difference in recurrence } \\
\text { and incontinence with AF }\end{array}$ \\
\hline $\begin{array}{l}\text { Madbouly } \\
\text { et al. [20] }\end{array}$ & LIFT & $\begin{array}{l}\text { Advancement } \\
\text { flap }\end{array}$ & $\begin{array}{l}\text { Operative time: LIFT } 22.6 \mathrm{~min}, \mathrm{AF} \\
36.5 \mathrm{~min}, p=0.0001 \\
\text { Pain scores at } 1 \text { week: } 4.8 \pm 2.8 \mathrm{LIFT} \\
3.1 \pm 1.3, p=0.002\end{array}$ & & $\begin{array}{l}\text { In high trans-sphincteric } \\
\text { fistulas, LIFT and AF have } \\
\text { similar healing rate, } \\
\text { recurrences, continence, QOL } \\
\text { LIFT has less pain }\end{array}$ \\
\hline $\begin{array}{l}\text { Ho } \\
\text { et al. [30] }\end{array}$ & $\begin{array}{l}\text { Fistulotomy with } \\
\text { marsupialization }\end{array}$ & Fistulectomy & $\begin{array}{l}\text { Mean operating time: C } 28.47 \pm 6.7 \\
\text { min, I } 29.2 \pm 8.4 \text {, ns } \\
\text { Mean wound size: C } 2.4 \pm 0 \mathrm{~cm}^{2}, \mathrm{I} \\
1.2 \pm 0.1 \mathrm{~cm}^{2}, p=0.542 \\
\text { Duration of discharge: C } 4.3 \pm 1.4 \mathrm{wk} \\
\text { I } 2.6 \pm 1.2 \mathrm{wk}, p=0.012 \\
\text { Pain mean VAS higher in I but ns }\end{array}$ & $\begin{array}{l}\text { Infection rate: C } 32.6 \%, \mathrm{I} \\
34.9 \% \text {, ns } \\
\text { LOS mean: C } 3.9 \pm 0.9 \mathrm{~d}, \mathrm{I} \\
4.2 \pm 1.6 \mathrm{~d} \\
\text { Mean healing time: C } \\
36.4 \pm 12.8 \mathrm{~d}, \mathrm{I} 28.6 \pm 16.3 \mathrm{~d}, \\
p=0.002\end{array}$ & $\begin{array}{l}\text { Marsupialization significantly } \\
\text { heals wound quicker and may } \\
\text { improve anal continence by } \\
\text { preserving anal squeeze } \\
\text { pressures with the } \\
\text { disadvantage of lengthening } \\
\text { surgery time }\end{array}$ \\
\hline $\begin{array}{l}\text { Sahaki- } \\
\text { trungruang } \\
\text { et al. [29] }\end{array}$ & $\begin{array}{l}\text { Fistulotomy with } \\
\text { marsupialization }\end{array}$ & Fistulectomy & $\begin{array}{l}\text { Mean operating time: C } 28 \pm 6.35 \mathrm{~min} \text {, } \\
\text { I } 28.2 \pm 6.57, p=0.92, \mathrm{~ns} \\
\text { Mean wound size: C } 2.06 \pm 1.90 \mathrm{~cm}^{2} \\
\text { I } 1.23 \pm 0.87 \mathrm{~cm}^{2}, \mathrm{~ns}\end{array}$ & $\begin{array}{l}\text { Duration of discharge: } \mathrm{C} \\
4.1 \pm 1.9 \mathrm{wk}, \mathrm{I} 2.75 \pm 1.71 \mathrm{wk}, \\
p=0.035 \\
\text { Pain: no diff in pain scores. } \\
\text { Mean healing time: C } \\
6.75 \pm 1.83 \mathrm{wk}, \mathrm{I} 4.85 \pm 1.39 \mathrm{wk}, \\
p=0.003\end{array}$ & $\begin{array}{l}\text { No significant difference in } \\
\text { pain scores and complications } \\
\text { although sig diff in pethidine } \\
\text { usage suggests the advantage } \\
\text { of marsupialization in } \\
\text { reducing acute pain post- } \\
\text { operatively }\end{array}$ \\
\hline $\begin{array}{l}\text { Pescatori } \\
\text { et al. [28] }\end{array}$ & $\begin{array}{l}\text { Fistulotomy/ } \\
\text { fistulectomy with } \\
\text { marsupialization }\end{array}$ & $\begin{array}{l}\text { Fistulotomy/ } \\
\text { fistulectomy }\end{array}$ & $\begin{array}{l}\text { Median operative time for } \\
\text { marsupialization }=8 \mathrm{~min} \\
\text { No significant difference between } \\
\text { both groups VAS pain score after } 12 \\
\text { and } 24 \mathrm{~h} \\
\text { Bleeding rate: C } 46 \% \text {, I } 36 \%, p<0.05\end{array}$ & $\begin{array}{l}\text { Sepsis rate: C } 23 \%, \mathrm{I} 14 \%, \mathrm{~ns} \\
\text { Wound size: I } 1,749 \pm 66 \mathrm{~mm}^{2} \\
\text { to } 819 \pm 38 \mathrm{~mm}^{2}, p<0.001 \text {, to } \\
217 \pm 15 \mathrm{~mm}^{2} \text { at } 4 \text { wks, } p<0.01 \text {; } \\
\text { C } 1171 \pm 31 \mathrm{~mm}^{2} \text { to } 543 \pm 19 \\
\mathrm{~mm}^{2} \text { at } 4 \text { wks, ns } \\
\text { Six in each group had } \\
\text { incontinence post-op: } 2 \text { in each } \\
\text { new }\end{array}$ & $\begin{array}{l}\text { Marsupialization significantly } \\
\text { decreased wound size and risk } \\
\text { of bleeding. No increase in } \\
\text { sepsis or pain }\end{array}$ \\
\hline $\begin{array}{l}\text { Chalya } \\
\text { et al. [31] }\end{array}$ & $\begin{array}{l}\text { Fistulotomy + } \\
\text { marsupialization }\end{array}$ & Fistulotomy & $\begin{array}{l}\text { I: faster healing } 6 \cdot 0(0 \cdot 4) \text { versus } 10 \cdot 0 \\
(0 \cdot 5) \text { wks for C group; } p 0 \cdot 001 \\
\text { Two marsupialized wounds ( } 4 \\
\text { percent) broke down at follow-up, } \\
\text { only } 1 \text { ( } 2 \text { percent) patient in the I } \\
\text { group complained of incontinence } \\
\text { (to liquid stools and flatus) compared } \\
\text { with } 6 \text { ( } 12 \text { percent) in the C group, ns }\end{array}$ & $\begin{array}{l}\text { Recurrence: I } 1 / 51, \text { LO } 2 / 52 \\
\text { Operative time: I } 10(0 \cdot 7) \\
\text { versus } 8 \cdot 0(0 \cdot 5) \text { min for C } \\
\text { group; } p 0 \cdot 05 \text {. The } \\
\text { postoperative hospital stay was } \\
\text { similar between the } 2 \text { groups } \mathrm{C} \\
2 \cdot 0(0 \cdot 2) \text { days; I } 1 \cdot 0(0 \cdot 1) \text { days } \\
\text { Manometry: significant drop in } \\
\text { MSP at } 3 \text { months in the C } \\
\text { group compared with the I } \\
\text { group; } p 0.05\end{array}$ & $\begin{array}{l}\text { Significantly shorter healing } \\
\text { time and duration of wound } \\
\text { discharge with } \\
\text { marsupialization }\end{array}$ \\
\hline $\begin{array}{l}\text { Jain } \\
\text { et al. [32] }\end{array}$ & $\begin{array}{l}\text { Fistulotomy with } \\
\text { marsupialization }\end{array}$ & Fistulotomy & $\begin{array}{l}\text { Healing time: } \mathrm{I} 4.85 \pm 1.39 \text { wks, } \mathrm{C} \\
6.75 \pm 1.83, p=0.003 \\
\text { Operating time }- \text { no difference } \\
\text { between groups } \\
\text { Time to cessation of ooze: I } 2.75 \pm 1.71 \\
\text { wks, C } 4.10 \pm 1.91, p=0.035\end{array}$ & $\begin{array}{l}\text { Wound size: I } 1.23 \pm 0.87 \mathrm{~cm}^{2} \text {, } \\
\text { C } 2.06 \pm 1.90 \mathrm{~cm}^{2}, \mathrm{~ns} \\
\text { No difference in QOL } \\
\text { measures between both groups }\end{array}$ & $\begin{array}{l}\text { Significantly shorter healing } \\
\text { time and duration of wound } \\
\text { discharge with } \\
\text { marsupialization, no increase } \\
\text { in OT time }\end{array}$ \\
\hline $\begin{array}{l}\text { Nazeer } \\
\text { et al. [26] }\end{array}$ & $\begin{array}{l}\text { Fistulotomy (lay } \\
\text { open) }\end{array}$ & Fistulectomy & Median healing time: I 34 d, C 41 d & $\begin{array}{l}\text { Recurrence: I } 3 / 24, \mathrm{C} 2 / 21, n= \\
5 \text { needed decisional surgery (I } \\
2 \text {; C } 3 \text { ) before healing, } \\
\text { exclusion of these still resulted } \\
\text { in sig shorter healing time in I }\end{array}$ & $\begin{array}{l}\text { Fistulotomy gave shorter } \\
\text { LOS, less pain, shorter healing } \\
\text { time }\end{array}$ \\
\hline
\end{tabular}


Table 3 (continued)

\begin{tabular}{|c|c|c|c|c|c|}
\hline Study & $\begin{array}{l}\text { Procedures } \\
\text { compared }\end{array}$ & $\begin{array}{l}\text { Control } \\
\text { group }\end{array}$ & Results & & Main findings \\
\hline $\begin{array}{l}\text { Kronborg } \\
\text { et al. [27] }\end{array}$ & Fistulotomy & Fistulectomy & $\begin{array}{l}\text { LOS: I } 2 \text { d; C } 3.5 \mathrm{~d} \\
\text { Pain (analgesics, } n \text { ): I 9; C } 15\end{array}$ & $\begin{array}{l}\text { Bleeding: I 1; C } 5 \\
\text { Healing time: I } 38 \text { d; C } 40 \text { d }\end{array}$ & $\begin{array}{l}\text { Shorter healing time in } \\
\text { fistulotomy group, need study } \\
\text { to compare with excision with } \\
\text { primary suture }\end{array}$ \\
\hline $\begin{array}{l}\text { Filingeri } \\
\text { et al. [37] }\end{array}$ & $\begin{array}{l}\text { Radiofrequency } \\
\text { fistulotomy }\end{array}$ & Fistulotomy & $\begin{array}{l}\text { Op time mean: I } 18.3 \mathrm{~min}, \mathrm{C} 17.9 \\
\text { min, } p=0.05 \\
\text { Pain VAS day } 1 \text { mean: I } 2.8, \mathrm{C} 4.1 \text {, } \\
p=0.05\end{array}$ & $\begin{array}{l}\text { Healing time mean: I } 3.5 \mathrm{wks} \text {, } \\
\text { C } 5.9 \text { wks, } p=0.025 \text {; no } \\
\text { complications, no recurrence } \\
\text { (new anterior fistula not } \\
\text { recorded as recurrence) }\end{array}$ & $\begin{array}{l}\text { Radiofrequency gives less } \\
\text { post-op pain and faster } \\
\text { wound healing }\end{array}$ \\
\hline $\begin{array}{l}\text { Gupta } \\
\text { et al. [38] }\end{array}$ & $\begin{array}{l}\text { Radiofrequency } \\
\text { fistulotomy }\end{array}$ & Fistulotomy & $\begin{array}{l}\text { Average time of procedure: C } 37 \mathrm{~min} \text {, } \\
\text { I } 22 \mathrm{~min}, p 0.001 \\
\text { Average bleeding during procedure: } \\
\mathrm{C} 134 \mathrm{~mL}, \mathrm{I} 47 \mathrm{~mL}, p 0.0002 \\
\text { Intensity of postoperative pain (first } \\
24 \mathrm{~h} \text { ) VAS: C } 2-5 \text {, I } 0-3, p 0.2995 \\
\text { Period of postoperative pain: C } 9 \\
\text { days, I } 5 \text { days, } p 0.0121 \\
\text { Period of hospital stay: C } 56 \mathrm{~h}, \mathrm{I} 37 \mathrm{~h} \text {, } \\
p 0.0015\end{array}$ & $\begin{array}{l}\text { Impairment of continence for } \\
\text { flatus (patients, } n \text { ): C } 6, \text { I } 2, p \\
0.004 \\
\text { Time off work: C } 11 \text { days, I7 } \\
\text { days; } p 0.0121 \\
\text { Average healing time: C } 64 \\
\text { days, I } 47 \text { days, } p \text { 0.0009 } \\
\text { Recurrence or failure: C } 3 \text {, I } 1 \text {, } \\
p 0.0198\end{array}$ & $\begin{array}{l}\text { Radiofrequency fistulotomy } \\
\text { sig quicker with less bleeding } \\
\text { than conventional with less } \\
\text { pain and faster healing } \\
\text { without compromising } \\
\text { recurrence rates }\end{array}$ \\
\hline $\begin{array}{l}\text { Gupta } \\
\text { et al. [39] }\end{array}$ & $\begin{array}{l}\text { Radiofrequency } \\
\text { fistulotomy }\end{array}$ & Fistulotomy & $\begin{array}{l}\text { Average time of procedure: C } 41 \mathrm{~min} \text {, } \\
\text { I } 24 \text { min, } p 0.001 \\
\text { Average bleeding during procedure: } \\
\text { C } 84 \mathrm{~mL} \text {, I } 47 \mathrm{~mL}, p 0.0004 \\
\text { Period of postoperative pain: C } 9 \\
\text { days, I } 5 \text { days, } p 0.029 \\
\text { Period of hospital stay: C } 36 \mathrm{~h}, \mathrm{I} 21 \mathrm{~h} \text {, } \\
p 0.0022\end{array}$ & $\begin{array}{l}\text { Impairment of continence for } \\
\text { flatus: C } 12 \%, \mathrm{I} 4 \%, p 0.0077 \\
\text { Time off work: C } 11 \text { days, I7 } \\
\text { days, } p 0.029 \\
\text { Wound healing time: C } 64 \\
\text { days, I } 47 \text { days, } p 0.0017 \\
\text { Recurrence or failure: C } 1, \mathrm{I} 0 \text {, } \\
\text { ns }\end{array}$ & $\begin{array}{l}\text { Radiofrequency fistulotomy } \\
\text { had significantly shorter } \\
\text { procedure time and wound } \\
\text { healing, less bleeding, and } \\
\text { patients returned to work } \\
\text { sooner }\end{array}$ \\
\hline $\begin{array}{l}\text { Indian } \\
\text { Council of } \\
\text { Medical } \\
\text { Research [24] }\end{array}$ & $\begin{array}{l}\text { Kshaarasoota seton } \\
\text { ] }\end{array}$ & Fistulectomy & $\begin{array}{l}\text { Time to healing: at } 12 \text { wks: I } 68 \% \\
\text { healed, C } 89 \% \text {; at } 40 \text { wks: I } 92 \%, \text { C } \\
98 \% \\
\text { Time to healing varied according to } \\
\text { fistula type (In wks): subcut: I 5, C } 2 \text { : } \\
\text { low: I 8, C } 4.5 \text {; high: I 15, C } 8\end{array}$ & $\begin{array}{l}\text { Incontinence at } 1 \text { year: I 8, C } \\
13 \\
\text { Impact of previous surgery on } \\
\text { recurrence: I } 6 \text { versus } 3 \% \text {, C } 24 \\
\text { versus } 7 \% \text {; recurrence: I 6/155, } \\
\text { C } 16 / 142\end{array}$ & $\begin{array}{l}\text { Medicated seton } \\
\text { (Kshaarasootra) offers an } \\
\text { effective and safe alternative } \\
\text { for the treatment of perianal } \\
\text { fistulas with better recurrence } \\
\text { rates than fistulectomy but } \\
\text { initial healing time is longer }\end{array}$ \\
\hline $\begin{array}{l}\text { Zbar } \\
\text { et al. [22] }\end{array}$ & $\begin{array}{l}\text { IAS preserving } \\
\text { seton }\end{array}$ & Cutting seton & $\begin{array}{l}\text { Incontinence }=\text { I } n=1 \text { (flatus), } \\
\text { C } n=2 \text { ( } 1 \text { flatus and } 1 \text { faecal leakage) } \\
\text { Recurrence: I } 2 / 18, \text { C } 1 / 16 \\
\text { Healing time: (mean) I } 14 \text { wks, C } 12 \\
\text { wks }\end{array}$ & $\begin{array}{l}\text { No preoperative differences } \\
\text { between patients and controls } \\
\text { in resting manometric } \\
\text { parameters and squeeze } \\
\text { parameters. Difference AUIC } \\
\text { of rectoinhibitory reflex at all } \\
\text { levels between } 2 \text { operative } \\
\text { groups significant } p<0.05\end{array}$ & $\begin{array}{l}\text { No difference in post- } \\
\text { operative continence scores, } \\
\text { recurrence, and healing times } \\
\text { between IAS preserving seton } \\
\text { and cutting seton. Need for a } \\
\text { larger prospective RCT to } \\
\text { evaluate }\end{array}$ \\
\hline $\begin{array}{l}\text { Lu } \\
\text { et al. [21] }\end{array}$ & Cutting seton & $\begin{array}{l}\text { Fistulotomy } \pm \\
\text { draining }\end{array}$ & $\begin{array}{l}\text { Healing time in days: (high) I } \\
24.73 \pm 8.15, \mathrm{C} 32.20 \pm 12.6, p<0.01 \text {; } \\
\text { (low) I } 22.26 \pm 8.67, \mathrm{C} 31.41 \pm 11.39 \\
p<0.01 \\
\text { Healing rate: High I } 54 / 56, \mathrm{C} 53 / 53 \text {; } \\
\text { low I 63/64, C } 61 / 63 \text {; not significant } \\
\text { between groups } \\
\text { No difference in integral calculus } \\
\text { pain scores, and QOL scores }\end{array}$ & $\begin{array}{l}\text { Confidence in treatment scores } \\
\text { better in I than } C, p<0.05 \\
\text { Hospital expenses significantly } \\
\text { lower in I, } p<0.01 \\
\text { Max anal squeeze signif lower } \\
\text { in controls } \\
\text { Anal sphincter function at } 3 \\
\text { month f/u less than C both in } \\
\text { low } p=0.03 \text { and high } 0.02\end{array}$ & $\begin{array}{l}\text { Benefits of thread dragging } \\
\text { through fistula method in } \\
\text { simple fistulas: Shorter } \\
\text { course, lower cost, better } \\
\text { QOL, and protects anal } \\
\text { function }\end{array}$ \\
\hline
\end{tabular}

LIFT, ligation of intersphincteric fistula tract; IAS, internal anal sphincter. 


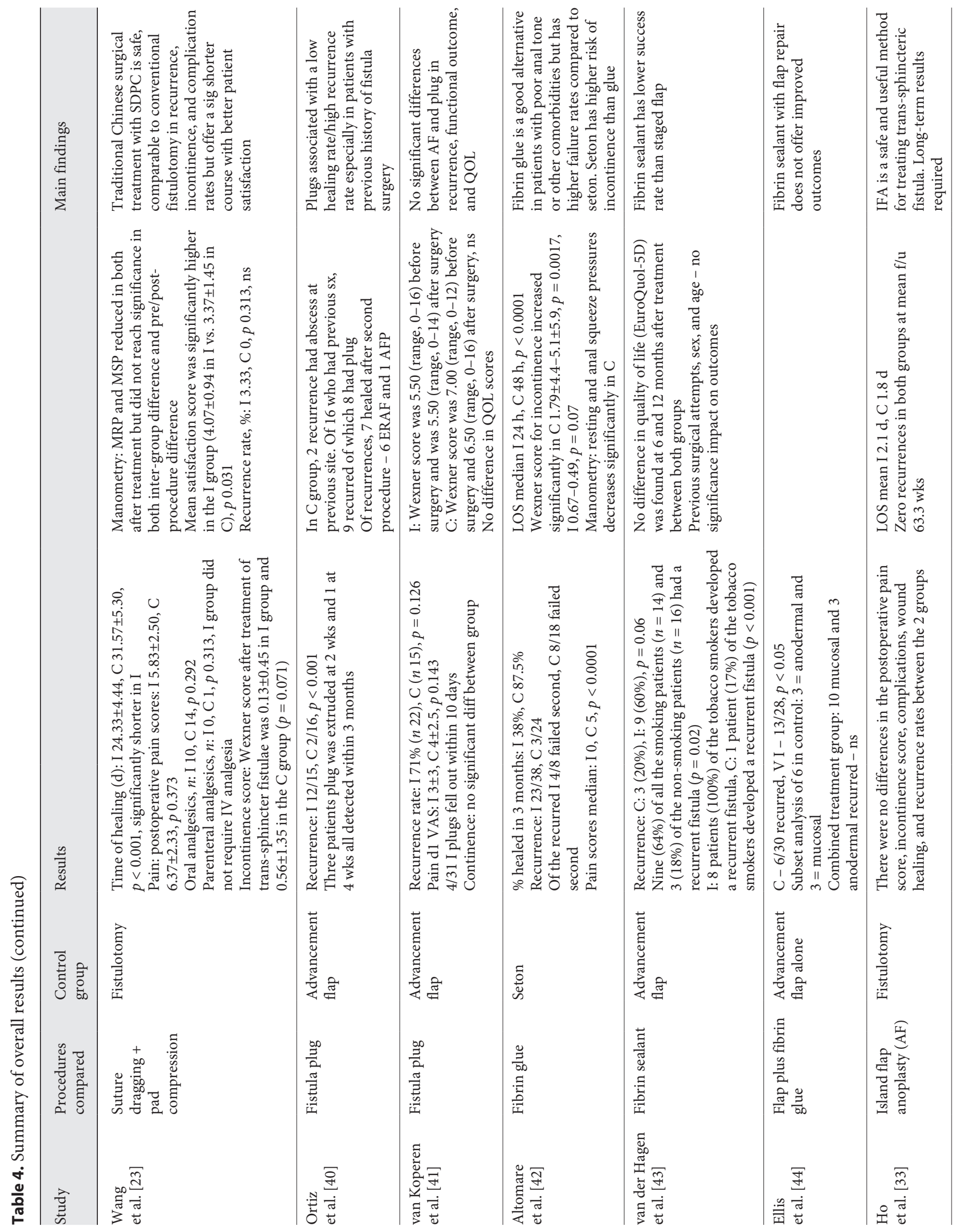




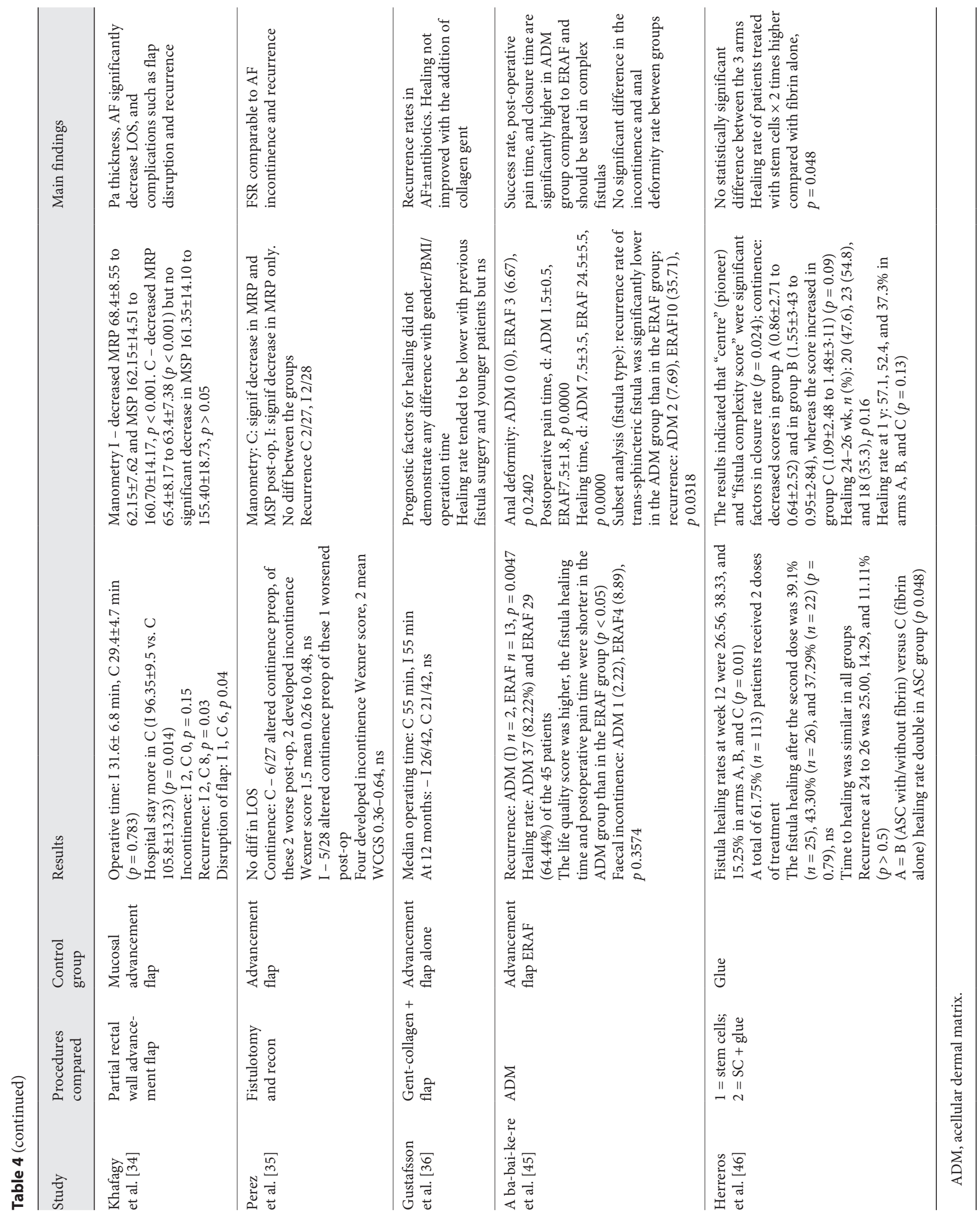


tori et al. [28] generated an OR of 1.11 for recurrence (95\% CI: 0.02-6.15, $p=0.91$; online suppl. Fig. 3 ) and an OR for incontinence of 1.10 (95\% CI: 0.14-8.56, $p=0.93$; online suppl. Fig. 3).

\section{Advancement Flaps}

Four studies included in our review examined advancement flaps [33-36]. Meta-analysis was not possible due to a single RCT. Island flap anoplasty was compared with fistulotomy. Sample size was small, and the authors reported no recurrence. Incontinence rates between island flap anoplasty and fistulotomy were also not significant (OR 0.64, 95\% CI: $0.10-4.10, p=0.64$ ). Khafagy et al. [34] compared partial rectal wall advancement flap to conventional mucosal advancement flap. They found that recurrence is lower in the partial rectal wall advancement flap group with an OR of 0.17 (95\% CI: 0.03-0.92, $p=$ $0.04)$. Incontinence rates are comparable between partial rectal wall advancement flap and conventional mucosal advancement flap with no significant difference (OR 5.54, 95\% CI: 0.25-123.08, $p=0.28$ ). Perez et al. [35] reported on recurrence rates from the comparison of fistulotomy with sphincter reconstruction with advancement flap [35]. There was no significant difference in recurrence on incontinence rates (recurrence: OR 0.96, 95\% CI: 0.13$7.36, p=0.97$; incontinence: OR 2.0, 95\% CI: 0.33-12.55, $p=0.45)$.

\section{Radiofrequency Fistulotomy versus Conventional Fistulotomy}

Three studies included examined radiofrequency fistulotomy versus conventional fistulotomy [37-39]. Analysis of pooled data gave an OR for recurrence of 0.33 (95\% CI: $0.05-2.14, p=0.24)$. There is no evidence of significant heterogeneity between groups $\left(I^{2} 0 \%\right)$. There was no significant difference in recurrence rates (online suppl. Fig. 4a). There were improved post-operative incontinence rates in the radiofrequency group. This did not reach statistical significance in pooled data analysis. OR for incontinence is 0.31 (95\% CI: $0.08-1.21, p=0.09$; online suppl. Fig. 4b) $\left(I^{2}=0 \%\right)$.

\section{Fistula Plugs}

Two studies compared fistula plug with advancement flap as a control group [40,41] and found a higher recurrence rate in the plug group. In the study by Ortiz et al. [40] recruitment stopped after 3 months due to high recurrence rate in the plug arm at preliminary analysis. Van Koperen et al. [41] showed no difference between recurrence rates which were high in both groups. Pooled data analysis on recurrence showed an OR of 4.22 favouring advancement flap (95\% CI: 1.76-10.13, $p=0.03$; online suppl. Fig. 5). They reported no difference in incontinence scores in either groups.

\section{Fibrin Glue}

Three studies compared the use of fibrin glue with 3 different controls: seton, advancement flaps, and glue as an adjunct to flaps versus flap alone [42-44]. Altomare et al. [42] compared fibrin glue with the seton group (cutting or loose). Recruitment was stopped after 3 months as preliminary data showed clear advantage in the control arm. A minimum follow-up period of 1 year was noted. The authors found that the glue group had significantly increased recurrence (23/38 in the glue group vs. $3 / 24$ in the seton group, OR 10.73, 95\% CI: $2.72-42.39, p=$ $0.0007)$. There was an increase in incontinence from $6 / 24$ to $15 / 24$ in the seton group at completion of follow-up $(p=0.0017)$. Van der Hagen et al. [43] compared glue with advancement flap. Recruitment stopped after a total of 30 patients were enrolled due to high recurrence rate in the glue arm. Nine out of 15 of the glue group recurred versus 3 out of 15 in control (OR 6.00, 95\% CI: 1.17-30.72, $p=0.03)$.

\section{Others}

\section{Acellular Dermal Matrix versus ERAF}

A novel bioprosthetic matrix used to close the fistula tracts was compared to endorectal advancement flap [45]. This study showed promising results for this bioprosthetic acellular dermal matrix (J-I ADM; J.Y. Life Tissue Engineering Co., Ltd., China) with a recurrence rate of 2 out of 45 compared to 13 out of 45 in the advancement flap control group (OR 0.11, 95\% CI: $0.02-0.54, p=0.006$ ). There was no significant difference in incontinence (OR $0.23,95 \%$ CI: $0.02-2.17, p=0.2$ ).

\section{Stem Cells}

Stem cells in the treatment of perianal fistulas were investigated in a single blinded multi-centred clinical trial with 3 parallel groups: stem cells alone, stem cell + fibrin glue, and fibrin glue alone [46]. This study did not show any significant difference in healing rates, recurrence, and incontinence rates between the 3 groups. The healing rate was 2 times higher in the stem cell group (RR 2.039, 95\% CI: $1-4.15, p=0.048$ ).

Video-Assisted Anal Fistula Treatment

Video-assisted anal fistula treatment is another novel treatment that shows some promise. It is unclear which 
part of the treatment is important and whether the video element is vital to its success or whether thorough cleaning of the track and secure closure of the internal opening is equally beneficial $[47,48]$. Success rates of $>75 \%$ fistula healing with no impact upon continence have been reported in single series.

\section{Discussion}

Perianal fistulas form a significant part of the workload of a colorectal surgeon. While there are many different types of treatment, there is no current gold standard. Clinicians tend to utilize one treatment or another depending on their own anecdotal experiences. It is a very distressing condition for the patient and can have a very negative impact on the patients' quality of life. A Cochrane review in 2010 concluded that there is no major difference between the various techniques with regards to recurrence rates, and fibrin glue and advancement flaps have lower incontinence rates [15]. At that time, evidence for the LIFT procedure was not yet available. Since then, the literature pertaining to anal fistula has expanded significantly. With this in mind, we embarked on the current study to establish the optimal surgical intervention for idiopathic (cryptoglandular) perianal fistula.

Recurrence rates ranging from 0 to $80 \%$ have been reported across all the examined surgical techniques. Many comparative groups have a single randomized control trial, and hence meta-analysis was not possible. Despite updates on newer interventions, we found that in terms of recurrence, the majority of interventions showed no significant difference compared to a specific control treatment. Our review shows that although the LIFT procedure has a reported success rate of $70 \%$ [47], there is no statistically significant difference in both recurrence and incontinence rates when compared with advancement flap.

Early results for fibrin plugs report favourable success rates of up to $80 \%$, and it was postulated that it could reduce overall recurrence. A multi-centre randomized control trial (PLUG Trial) found no difference in recurrence rates between fibrin plugs and advancement flaps. However, when we pooled data incorporating the results of the PLUG Trial, meta-analysis of 2 studies showed that fistula plugs have significantly higher recurrence rates when compared to advancement flaps with an odds ratio of 4.22 favouring advancement flap (95\% CI: 1.76-10.13, $p=$ 0.03; online suppl. Fig. 5) [49]. We also found no significant difference in overall incontinence rates.

Surgical Management of Perianal Fistulas
The other most commonly reported outcomes in the included studies are pain, healing time, and operative time. Pain scores were found to be reduced in novel treatments such as the LIFT procedure and acellular dermal matrix. Radiofrequency fistulotomy is reported to have less pain scores and reduced bleeding. This has been postulated to be due to the coagulation of blood vessels and sealing of nerves by radiofrequency waves.

Having considered the evidence including the novel (previously not evaluated) interventions, our results mirror those of the Cochrane 2010 review and that there is no difference in recurrence between the majority of interventions. However, fibrin glue and plugs show higher recurrence in the RCT setting. In the absence of favourable incontinence rates, these findings question the continual inclusion of glues and plugs in the armamentarium for the treatment of perianal fistulas. Without further evidence, from the results of this study, we are unable to make specific recommendations regarding a more robust treatment algorithm for the management of perianal fistulas. We postulate that specific treatments applied to a specific fistula subtype may generate more favourable outcomes, but we await with interest the outcomes of future trials to support this hypothesis.

With ongoing evidence that surgical interventions have no difference in terms of recurrence and incontinence, it may be useful to analyze these outcomes in different subgroups, for example, female gender, anterior fistulas, and recurrent fistulas, that is, high risk for incontinence or recurrence groups, in order to develop more patient-specific treatment guidelines rather than looking for a single best fit for all. This may be difficult as numbers in these specific subgroups are likely low and therefore difficult to perform a well-powered study. Multi-centre international-based collaborative RCTs may generate sufficient patient numbers and follow-up duration to facilitate this subset analysis.

The main strength of our review is our exhaustive search strategy which yielded a large number of eligible studies. All studies included are randomized control studies, which offer a high level of evidence (grade IB). We provided updated evidence on a range of novel interventions. This is a very broad topic with multiple comparison groups rather than a more conventional intervention versus control scenario. This is one of the major limitations as there was a limited number of RCTs available in each of the comparisons, and hence pooled data was not possible in all comparison groups. In many cases, results from 1 study are analyzed rather than full metaanalysis. Most of the studies are scored as low $(<2)$ or av- 
erage (3) on the Jadad quality assessment scale with a mean of 2.46, just below average for good-quality studies. Funnel plots could not show meaningful conclusions on bias due to the small number of studies in each comparison group, and shape of the funnel plot is not apparent (online suppl. material).

\section{Conclusion}

Current evidence failed to demonstrate an optimal surgical strategy for the management of non-Crohn's related perianal fistulas. There is a need for further assessment of the benefit of stem cells compared to other conventional treatments. In the interim, in absence of convincing evidence, it is likely that individual clinicians will continue to utilize treatment options based on patient factors, their personal experience, and often influenced by enthusiasm for novel as yet unproven therapies which require further evaluation within robust study designs.

\section{Acknowledgements}

We would like to acknowledge the help of the RCSI library in supporting our electronic searches.

\section{Statement of Ethics}

This meta-analysis was conducted in accordance with the Preferred Reporting Items for Systematic Reviews and Meta-Analyses (PRISMA) guideline. Approval from an ethics committee was not required for this study.

\section{Conflict of Interest Statement}

The authors have no conflicts of interest to declare.

\section{Funding Sources}

There was no funding.

\section{Author Contributions}

X.C. developed the search and wrote the initial draft. T.F. reviewed the methodology and thoroughly reviewed the manuscript. A.R. did the searches along with X.C. and reviewed the manuscript. J.P. reviewed the manuscript and provided suitable modifications which enhanced the manuscript. D.K. developed the idea, supported X.C. in the methods, and reviewed, submitted, and revised the manuscript.

\section{References}

1 Williams NS, O'Connell PR. The anus and anal canal. In: Williams NS, editor. Bailey \& Love's short practice of surgery. 25th ed. London: Hodder Arnold; 2008. p. 1240-72.

2 Hermann GDL. Sur la muquese de la region cloacale du rectum. C R Acad Sci. 1880;(90): $1301-2$.

3 Kelly ME, Heneghan HM, McDermott FD, Nason GJ, Freeman C, Martin ST, et al. The role of loose seton in the management of anal fistula: a multicenter study of 200 patients. Tech Coloproctol. 2014;18(10):915-9.

4 Akhtar M. Fistula in ano: an overview. JIMSA. 2012;25(1):53-5.

5 Hippocrates. On Fistulae. Available from: http: //classics.mit.edu//Hippocrates/fistulae. html.

6 Gupta PJ, Gupta SN, Heda PS. Which treatment for anal fistula? Cut or cover, plug or paste, loop or lift. Acta Chir Iugosl. 2012; 59(2):15-20.

7 Nicholls RJ. Fistula in ano: an overview. Acta Chir Iugosl. 2012;59(2):9-13.

8 Subhas G, Singh Bhullar J, Al-Omari A, Unawane A, Mittal VK, Pearlman R. Setons in the treatment of anal fistula: review of variations in materials and techniques. Dig Surg. 2012; 29(4):292-300.
9 Ritchie RD, Sackier JM, Hodde JP. Incontinence rates after cutting seton treatment for anal fistula. Colorectal Dis. 2009;11(6):56471.

10 Rojanasakul A, Pattanaarun J, Sahakitrungruang C, Tantiphlachiva K. Total anal sphincter saving technique for fistula-in-ano; the ligation of intersphincteric fistula tract. J Med Assoc Thai. 2007;90(3):581-6.

11 Ratto C, Grillo E, Parello A, Costamagna G Doglietto GB. Endoanal ultrasound-guided surgery for anal fistula. Endoscopy. 2005; 37(8):722-8.

12 Sentovich SM. Fibrin glue for anal fistulas: long-term results. Dis Colon Rectum. 2003; 46(4):498-502.

13 Sentovich SM. Fibrin glue for all anal fistulas. J Gastrointest Surg. 2001;5(2):158-61.

14 Garcia-Olmo D, Herreros D, Pascual I, Pascual JA, Del-Valle E, Zorrilla J, et al. Expanded adipose-derived stem cells for the treatment of complex perianal fistula: a phase II clinical trial. Dis Colon Rectum. 2009;52(1): 79-86.

15 Jacob TJ, Perakath B, Keighley MR. Surgical intervention for anorectal fistula. Cochrane Database Syst Rev. 2010;(5):CD006319.
16 Yassin NA, Askari A, Warusavitarne J, Faiz OD, Athanasiou T, Phillips RK, et al. Systematic review: the combined surgical and medical treatment of fistulising perianal Crohn's disease. Aliment Pharmacol Ther. 2014;40(7):741-9.

17 Google Translate. Available from: https:// translate.google.ie.

18 Jadad AR, Moore RA, Carroll D, Jenkinson C, Reynolds DJ, Gavaghan DJ, et al. Assessing the quality of reports of randomized clinical trials: is blinding necessary? Control Clin Trials. 1996;17(1):1-12.

19 Mushaya C, Bartlett L, Schulze B, Ho YH. Ligation of intersphincteric fistula tract compared with advancement flap for complex anorectal fistulas requiring initial seton drainage. Am J Surg. 2012;204(3):283-9.

20 Madbouly KM, El Shazly W, Abbas KS, Hussein AM. Ligation of intersphincteric fistula tract versus mucosal advancement flap in patients with high transsphincteric fistula-inano: a prospective randomized trial. Dis Colon Rectum. 2014;57(10):1202-8.

21 Lu JG, Cao YQ, He CM, Guo XT, Huang HX, Yi J, et al. Clinical research of thread-dragging through fistula method in treating patients with simple anorectal fistula. Zhong Xi Yi Jie He Xue Bao. 2006;4(2):140-6. 
22 Zbar AP, Ramesh J, Beer-Gabel M, Salazar R, Pescatori M. Conventional cutting vs. internal anal sphincter-preserving seton for high trans-sphincteric fistula: a prospective randomized manometric and clinical trial. Tech Coloproctol. 2003;7(2):89-94.

23 Wang C, Lu JG, Cao YQ, Yao YB, Guo XT, Yin HQ. Traditional Chinese surgical treatment for anal fistulae with secondary tracks and abscess. World J Gastroenterol. 2012; 18(40):5702-8.

24 Multicentric randomized controlled clinical trial of Kshaarasootra (Ayurvedic medicated thread) in the management of fistula-in-ano. Indian Council of Medical Research. Indian J Med Res. 1991;94:177-85.

25 Ho KS, Tsang C, Seow-Choen F, Ho YH, Tang CL, Heah SM, et al. Prospective randomised trial comparing ayurvedic cutting seton and fistulotomy for low fistula-in-ano. Tech Coloproctol. 2001;5(3):137-41.

26 Nazeer MA, Saleem R, Ali M, Ahmed ZN. Better option for the patients of low fistula in ano: fistulectomy or fistulotomy. Pak J Med Health Sci. 2012;6(4):885-7.

27 Kronborg O. To lay open or excise a fistulain-ano: a randomized trial. Br J Surg. 1985; 72(12):970

28 Pescatori M, Ayabaca SM, Cafaro D, Iannello A, Magrini S. Marsupialization of fistulotomy and fistulectomy wounds improves healing and decreases bleeding: a randomized controlled trial. Colorectal Dis. 2006;8(1):11-4.

29 Sahakitrungruang C, Pattana-Arun J, Khomvilai S, Tantiphlachiva $\mathrm{K}$, Atittharnsakul $\mathrm{P}$, Rojanasakul A. Marsupialization for simple fistula in ano: a randomized controlled trial. J Med Assoc Thai. 2011;94(6):699-703.

30 Ho YH, Tan M, Leong AF, Seow-Choen F. Marsupialization of fistulotomy wounds improves healing: a randomized controlled trial. Br J Surg. 1998;85(1):105-7.

31 Chalya PL, Mabula JB. Fistulectomy versus fistulotomy with marsupialisation in the treatment of low fistula-in-ano: a prospective randomized controlled trial. Tanzan J Health Res. 2013;15(3):193-8.
32 Jain BK, Vaibhaw K, Garg PK, Gupta S, Mohanty D. Comparison of a fistulectomy and a fistulotomy with marsupialization in the management of a simple anal fistula: a randomized, controlled pilot trial. J Korean Soc Coloproctol. 2012;28(2):78-82.

33 Ho KS, Ho YH. Controlled, randomized trial of island flap anoplasty for treatment of transsphincteric fistula-in-ano: early results. Tech Coloproctol. 2005;9(2):166-8.

34 Khafagy W, Omar W, El Nakeeb A, Fouda E, Yousef M, Farid M. Treatment of anal fistulas by partial rectal wall advancement flap or mucosal advancement flap: a prospective randomized study. Int J Surg. 2010;8(4):321-5.

35 Perez F, Arroyo A, Serrano P, Sánchez A, Candela F, Perez MT, et al. Randomized clinical and manometric study of advancement flap versus fistulotomy with sphincter reconstruction in the management of complex fistula-in-ano. Am J Surg. 2006;192(1):34-40.

36 Gustafsson UM, Graf W. Randomized clinical trial of local gentamicin-collagen treatment in advancement flap repair for anal fistula. Br J Surg. 2006;93(10):1202-7.

37 Filingeri V, Gravante G, Baldessari E, Casciani CU. Radiofrequency fistulectomy vs. diathermic fistulotomy for submucosal fistulas: a randomized trial. Eur Rev Med Pharmacol Sci. 2004;8(3):111-6.

38 Gupta PJ. Radiosurgical fistulotomy; an alternative to conventional procedure in fistula in ano. Curr Surg. 2003;60(5):524-8.

39 Gupta PJ. Anal fistulotomy with radiofrequency: a better option to a conventional procedure. Rom J Gastroenterol. 2003;12(4): 287-91.

40 Ortiz H, Marzo J, Ciga MA, Oteiza F, Armendáriz P, de Miguel M. Randomized clinical trial of anal fistula plug versus endorectal advancement flap for the treatment of high cryptoglandular fistula in ano. Br J Surg. 2009; 96(6):608-12.
41 van Koperen PJ, Bemelman WA, Gerhards MF, Janssen $L W$, van Tets WF, van Dalsen $A D$, et al. The anal fistula plug treatment compared with the mucosal advancement flap for cryptoglandular high transsphincteric perianal fistula: a double-blinded multicenter randomized trial. Dis Colon Rectum. 2011;54(4):387-93.

42 Altomare DF, Greco VJ, Tricomi N, Arcanà F Mancini S, Rinaldi M, et al. Seton or glue for trans-sphincteric anal fistulae: a prospective randomized crossover clinical trial. Colorectal Dis. 2011;13(1):82-6.

43 van der Hagen SJ, Baeten CG, Soeters PB, van Gemert WG. Staged mucosal advancement flap versus staged fibrin sealant in the treatment of complex perianal fistulas. Gastroenterol Res Pract. 2011;2011:186350.

44 Ellis CN, Clark S. Fibrin glue as an adjunct to flap repair of anal fistulas: a randomized, controlled study. Dis Colon Rectum. 2006;49(11): 1736-40.

45 A ba-bai-ke-re M-M-T-J, Wen H, Huang HG, Chu H, Lu M, Chang ZS, et al. Randomized controlled trial of minimally invasive surgery using acellular dermal matrix for complex anorectal fistula. World J Gastroenterol. 2010; 16(26):3279-86

46 Herreros MD, Garcia-Arranz M, Guadalajara H, De-La-Quintana P, Garcia-Olmo D. Autologous expanded adipose-derived stem cells for the treatment of complex cryptoglandular perianal fistulas: a phase III randomized clinical trial (FATT 1: fistula advanced therapy trial 1) and long-term evaluation. Dis Colon Rectum. 2012;55(7):762-72.

47 Zirak-Schmidt S, Perdawood SK. Management of anal fistula by ligation of the intersphincteric fistula tract: a systematic review. Dan Med J. 2014;61(12):A4977.

48 Meinero P, Mori L. Video-assisted anal fistula treatment (VAAFT): a novel sphincter-saving procedure for treating complex anal fistulas. Tech Coloproctol. 2011;15(4):417-22.

49 Williams G, Williams A, Tozer P, Phillips R, Ahmad A, Jayne D, et al. The treatment of anal fistula: second ACPGBI Position Statement: 2018. Colorectal Dis. 2018 Jul;20(Suppl 3):5-31. 